\title{
Balanced Excitation and Inhibition Determine Spike Timing during Frequency Adaptation
}

\author{
Michael J. Higley and Diego Contreras \\ Department of Neuroscience, University of Pennsylvania School of Medicine, Philadelphia, Pennsylvania 19104
}

\begin{abstract}
In layer 4 (L4) of the rat barrel cortex, a single whisker deflection evokes a stereotyped sequence of excitation followed by inhibition, hypothesized to result in a narrow temporal window for spike output. However, awake rats sweep their whiskers across objects, activating the cortex at frequencies known to induce short-term depression at both excitatory and inhibitory synapses within L4. Although periodic whisker deflection causes a frequency-dependent reduction of the cortical response magnitude, whether this adaptation involves changes in the relative balance of excitation and inhibition and how these changes might impact the proposed narrow window of spike timing in L4 is unknown. Here, we demonstrate for the first time that spike output in L4 is determined precisely by the dynamic interaction of excitatory and inhibitory conductances. Furthermore, we show that periodic whisker deflection results in balanced adaptation of the magnitude and timing of excitatory and inhibitory input to L4 neurons. This balanced adaptation mediates a reduction in spike output while preserving the narrow time window of spike generation, suggesting that L4 circuits are calibrated to maintain relative levels of excitation and inhibition across varying magnitudes of input.
\end{abstract}

Key words: barrels; whisker; intracellular; cortex; synaptic; conductance

\section{Introduction}

Cortical representation of sensory information across modalities is shaped by the local balance of excitation and inhibition (Kyriazi et al., 1996; Fox et al., 2003; Wehr and Zador, 2003; Zhang et al., 2003; Wilent and Contreras, 2004; Marino et al., 2005; Priebe and Ferster, 2005). In the rodent whisker system, ascending thalamic input engages neuronal circuits in cortical layer 4 (L4), consisting of excitatory spiny stellate and pyramidal cells as well as aspiny interneurons (White and Rock, 1981; Beierlein et al., 2002, 2003; Bruno and Simons, 2002) that provide feedforward and feedback inhibition to the local network (Agmon and Connors, 1991; Swadlow and Gusev, 2000; Porter et al., 2001; Swadlow, 2003; Staiger et al., 2004). This functional architecture leads to a precise sequence of excitation followed by inhibition in response to whisker deflection that may serve to sharpen the spike timing of suprathreshold responses and limit the time for integration of excitatory inputs (Pinto et al., 2000, 2003; Pouille and Scanziani, 2001; Wehr and Zador, 2003; Wilent and Contreras, 2004; Blitz and Regehr, 2005; Mittmann et al., 2005).

Most studies have examined the relationship between synaptic input and spike timing using single sensory stimuli. However, sensation is an active process that often involves repeated sampling over time (Ahissar and Arieli, 2001). Awake rats repeatedly sweep their whiskers across objects in the en-

Received Aug. 18, 2005; revised Nov. 4, 2005; accepted Nov. 10, 2005

This work was supported by the Human Frontier Science Program. We thank Dr. Jessica A. Cardin for helpful discussion on this manuscript and Dr. Esther Garcia de Yebenes for help with histological preparation.

Correspondence should be addressed to Diego Contreras, Department of Neuroscience, University of Pennsylvania School of Medicine, 215 Stemmler Hall, Philadelphia, PA 19104. E-mail: diegoc@mail.med.upenn.edu. DOI:10.1523/JNEUROSCI.3506-05.2006

Copyright $\odot 2006$ Society for Neuroscience $\quad$ 0270-6474/06/260448-10\$15.00/0 vironment at frequencies ranging from 5 to $20 \mathrm{~Hz}$ (Welker, 1964; Carvell and Simons, 1990), resulting in periodic firing of barrel cortex neurons in phase with the movement (Fee et al., 1997). Whisker-evoked suprathreshold responses exhibit frequency-dependent adaptation (Fanselow and Nicolelis, 1999; Ahissar et al., 2000, 2001; Garabedian et al., 2003; Castro-Alamancos, 2004; Khatri et al., 2004) that is at least partially dependent on thalamocortical synaptic depression (Chung et al., 2002). However, both excitatory and inhibitory corticocortical synapses within L4 also undergo frequencydependent short-term depression (Thomson and West, 1993; Petersen, 2002; Beierlein et al., 2003; Cowan and Stricker, 2004; Staiger et al., 2004), making it difficult to predict the net changes in excitation and inhibition after periodic whisker deflection. Using extracellular recordings, Khatri et al. (2004) found that putative excitatory and inhibitory units in L4 exhibited a similar magnitude of frequency adaptation. However, intracellular recordings are necessary to determine the relative postsynaptic changes in excitatory and inhibitory input.

In the present study, we show that, for L4 cells, whisker deflection evokes overlapping excitatory and inhibitory synaptic conductances that long outlast the duration of the suprathreshold response. The relative magnitude and timing of excitation and inhibition define a narrow window over which input integration and spike output can occur. Moreover, repetitive whisker deflection results in a balanced decrease in both excitatory and inhibitory inputs, reducing spike output while maintaining a narrow spike timing window. By exploring responses in a model L4 neuron, we also show that unbalanced adaptation of excitation and inhibition results in disruption of both the reliability and timing of L4 spike output. 


\section{Materials and Methods}

Surgery and preparation. Nineteen adult male Sprague Dawley rats (350$450 \mathrm{~g}$ ) were used in the present study. Animals were anesthetized with isoflurane (0.5-2.0\%), paralyzed with gallamine triethiodide, and artificially ventilated. End-tidal $\mathrm{CO}_{2}(3.5-3.7 \%)$ and heart rate were continuously monitored. Body temperature was maintained at $37^{\circ} \mathrm{C}$ via servocontrolled heating blanket and rectal thermometer. The depth of anesthesia was maintained by adjusting the percentage of inspired isoflurane to keep a low heart rate (250-300 beats/min) and constant highamplitude, low-frequency electroencephalogram as recorded from a bipolar tungsten electrode lowered into the cortex. The animal was placed in a stereotaxic apparatus, a craniotomy was made to expose the barrel cortex (1.0-3.0 $\mathrm{mm}$ posterior to bregma, $4.0-7.0 \mathrm{~mm}$ lateral to the midline), and the dura was resected. Recording stability was improved by drainage of the cisterna magna and filling of the craniotomy with a solution of $3.5 \%$ agar after electrode placement.

Electrophysiological recordings. Intracellular recordings were performed with glass micropipettes pulled on a P-97 Brown-Flaming puller (Sutter Instruments, Novato, CA). Pipettes were filled with $3 \mathrm{M}$ potassium acetate and $2.75 \%$ neurobiotin and had DC resistances of $60-80$ $\mathrm{M} \Omega$. For some recordings, $25 \mathrm{~mm}$ QX-314 (lidocaine $N$-ethyl bromide) was included in the recording pipette to block fast sodium action potentials. The recording pipette was lowered into the brain, oriented normal to the cortical surface. Vertical depth was read on the scale of the micromanipulator and verified histologically for recovered neurobiotin-filled cells (see Fig. 1d). A high-impedance amplifier (low-pass filter of $5 \mathrm{kHz}$ ) with active bridge circuitry (Cygnus Technology, Delaware Water Gap, PA) was used to record and inject current into cells. All recordings at resting $V_{\mathrm{m}}$ were made without holding current. Extracellular recordings were performed using glass-insulated tungsten electrodes with $1 \mathrm{kHz}$ impedance of 1.5 M $\Omega$ (Alpha-Omega, Alpharetta, GA). Extracellular signals were amplified and filtered from $500 \mathrm{~Hz}$ to $10 \mathrm{kHz}$ (FHC, Bowdoinham, ME). Intracellular and extracellular recordings were digitized at 10 and $20 \mathrm{kHz}$, respectively, and saved to a disk for off-line analysis.

Whisker stimulation. For each cell, the principal whisker (PW) was mechanically deflected in the dorsal direction using a ceramic piezoelectric bimorph stimulator (Piezo Systems, Cambridge, MA) as described previously (Simons, 1983; Higley and Contreras, 2003). Square electrical pulses ( $1 \mathrm{~ms}$ in duration) were applied to the stimulator resulting in a brief step-and-hold deflection of the whisker. This short duration pulse was chosen so that the "off response" would not interfere with the "on response" of the subsequent stimulus in a train. Although this fast deflection necessarily blurs the distinction between opposing directions, data from our laboratory suggest that the cortical response to this stimulus does not differ qualitatively or quantitatively from a high-velocity deflection in the same direction where the on and off responses are separated by $200 \mathrm{~ms}$. To study frequency adaptation, stimuli were applied at $10 \mathrm{~Hz}$ for a $1 \mathrm{~s}$ train ( 10 stimuli per train). A minimum of 30 trains, delivered at 0.25 $\mathrm{Hz}$, was used to generate data for each cell.

Data analysis. For spike analysis, well isolated single units extracted from extracellular records using a simple threshold algorithm were combined with suprathreshold responses from intracellular records, and peristimulus time histograms (PSTHs; bin size, $1 \mathrm{~ms}$ ) were constructed. The magnitude and latency of the spike responses were measured over a $0-20 \mathrm{~ms}$ poststimulus interval for each stimulus in a train. We further quantified the timing of spike output by calculating the $\mathrm{SD}(\sigma)$ and vector strength (VS) of the spike latencies over the same $20 \mathrm{~ms}$ interval. Although SD gives an absolute value of spike precision, vector strength is a normalized measure of the degree to which spikes occur at a given phase of the response window (here, $0-20 \mathrm{~ms}$ poststimulus) and ranges from 0 (all spike latencies equally represented) to 1 (all spikes occur at a single latency). VS was calculated similarly to previous studies (Garabedian et al., 2003; Khatri et al., 2004): $\mathrm{VS}=\mathrm{V}\left[\Sigma(\cos \theta)^{2}+\Sigma(\sin \theta)^{2}\right] / n$ and $\theta=$ $2 \pi(t / T)$, where $n$ is the total number of spikes, $t$ is the spike latency, and $T$ is the duration of the total response window $(20 \mathrm{~ms})$. Although the use of a $20 \mathrm{~ms}$ window differs from previous studies that used the entire interstimulus interval (100 ms), we felt it was important to use the same period for calculations of response magnitude and spike timing. Further- more, our values for VS (see Fig. 2D) did not differ appreciably from those of Khatri et al. (2004) using the longer interval.

For synaptic response analysis, spikes were removed by detecting spike threshold at the base of the action potential and extrapolating the $V_{\mathrm{m}}$ values from the start to the end of the spike, followed by smoothing with a three-point running average. Baseline membrane potential $\left(V_{\mathrm{m}}\right)$ was calculated as the mean $V_{\mathrm{m}}$ for the $10 \mathrm{~ms}$ preceding each stimulus in a train. Evoked postsynaptic potential (PSP) onset latency was defined as the first time point at which the $V_{\mathrm{m}}$ clearly deviated from baseline at the start of the response, and amplitude of the PSP was measured from baseline to the peak depolarization. The rate of rise for each PSP was measured by calculating the slope of the line connecting the points of 10 and $90 \%$ peak amplitude for each response. For all measures, values reported are the mean \pm SEM.

Calculation of synaptic conductances. For the cells in which fast sodium spikes were blocked using internal QX-314, the total membrane conductance of the cell was calculated at each time point immediately before and during a whisker-evoked PSP. We used the membrane equation: $C_{\mathrm{m}} \times$ $d V_{\mathrm{m}} / d t=-g_{\mathrm{T}}\left(V_{\mathrm{m}}-V_{\text {rev }}\right)+I_{\text {inj }}$, where $C_{\mathrm{m}}$ is the membrane capacitance of the cell, calculated by measuring the time constant from short hyperpolarizing current pulses, $g_{\mathrm{T}}$ is the total membrane conductance, $V_{\text {rev }}$ is the weighted combined reversal potential of all membrane conductances, and $I_{\text {inj }}$ is the injected current. This equation can be rewritten as: $V_{\mathrm{m}}=$ $1 / g_{\mathrm{T}} \times\left(I_{\text {inj }}-I_{\text {cap }}\right)+V_{\text {rev }}$, where $V_{\mathrm{m}}$ is a linear function of the injected current corrected for the capacitative current $\left(I_{\text {cap }}=C_{\mathrm{m}} \times d V_{\mathrm{m}} / d t\right)$. By evoking a synaptic response while holding the cell at multiple $V_{\mathrm{m}}$ levels using DC current injection, we could construct a $V-I$ plot (using the corrected $I$ value), where the inverse slope of the best-fit line was $g_{\mathrm{T}}$. By subtracting the value of $g_{\mathrm{T}}$ measured before the onset of the evoked response (effectively, the resting leak conductance) from the value of $g_{\mathrm{T}}$ at each point during the response, we derived a measurement of the total evoked synaptic conductance $\left(g_{\text {Syn }}\right)$ over time.

To decompose the total synaptic conductance, $g_{\text {Syn }}$, into excitatory and inhibitory components, we used the following simplification: $I_{\text {syn }}=$ $g_{\mathrm{E}}\left(V_{\mathrm{m}}-V_{\mathrm{E}}\right)+g_{\mathrm{I}}\left(V_{\mathrm{m}}-V_{\mathrm{I}}\right)$, where $I_{\text {syn }}$ is the total synaptic current, $g_{\mathrm{E}}$ and $g_{\mathrm{I}}$ are the total excitatory and inhibitory conductances, respectively, and $V_{\mathrm{E}}$ and $V_{\mathrm{I}}$ are the reversal potentials for excitation and inhibition, respectively. At the synaptic reversal potential, $V_{\text {rev }}, I_{\text {Syn }}=0$, resulting in: $0=g_{\mathrm{E}}\left(V_{\text {rev }}-V_{\mathrm{E}}\right)+g_{\mathrm{I}}\left(V_{\text {rev }}-V_{\mathrm{I}}\right)$. Using the simplification that $g_{\text {Syn }}=g_{\mathrm{E}}$ $+g_{\mathrm{I}}$, we obtain: $g_{\mathrm{I}}=g_{\text {Syn }}\left(V_{\mathrm{E}}-V_{\text {rev }}\right) /\left(V_{\mathrm{E}}-V_{\mathrm{I}}\right)$ and $g_{\mathrm{E}}=g_{\mathrm{Syn}}\left(V_{\mathrm{I}}-\right.$ $\left.V_{\text {rev }}\right) /\left(V_{\mathrm{E}}-V_{\mathrm{I}}\right) . V_{\text {rev }}$ can be calculated as the $y$ value of the intersection of the $V-I$ plot made at baseline with the $V-I$ plot made at each point in the synaptic response. Using the previous calculation of $g_{\text {Syn }}$ over time and assuming values of $0 \mathrm{mV}$ and $-80 \mathrm{mV}$ for $V_{\mathrm{E}}$ and $V_{\mathrm{I}}$, respectively, we can use these equations to obtain measures of the excitatory and inhibitory conductances as a function of time during the evoked response.

A potential source of error in the present analysis is the uncertainty in the assumption of values for synaptic reversal potentials, particularly in sharp electrode recordings where the internal solution is not concentration clamped. However, this uncertainty has only a minor quantitative and not qualitative effect on the results. In additional analyses (data not shown), we found that a $10 \mathrm{mV}$ change in $V_{\mathrm{I}}$ resulted in only a $15 \%$ change in the corresponding calculated conductance magnitude and no alteration of the conductance timing.

An additional source of error is the assumption of a linear, isopotential neuron inherent in the method of conductance calculation. The deviation of the actual membrane from these assumptions results in an underestimate of conductance magnitudes that is greater for inhibitory than excitatory inputs (Wehr and Zador, 2003). We have attempted to minimize the impact of this underestimate by only considering relative changes in conductance magnitude across trains of stimuli. In addition, theoretical work in the auditory system demonstrates that the relative timing of excitatory and inhibitory conductances is much less affected by the cable properties of the cell (Wehr and Zador, 2003), suggesting our calculations for the temporal aspects of whisker-evoked conductances are also valid.

Simulation. We used a single-compartment model developed by Destexhe et al. (2001) run in the NEURON simulation environment (Hines and Carnevale, 1997). Briefly, the model included Hodgkin and Huxley- 
type voltage-dependent sodium and potassium conductances to generate spikes. Realistic background activity was generated using two independent conductances for excitation and inhibition, simulated as one-variable stochastic processes (Destexhe et al., 2001). The average resting conductance of the model cell was $70 \mathrm{nS}$, and the membrane capacitance was $0.35 \mathrm{nF}$. The complete model can be obtained from http://cns.iaf.cnrs-gif.fr/alain_demos.html.

The sensory-evoked excitatory and inhibitory input conductances, $g_{\mathrm{E}}(t)$ and $g_{\mathrm{I}}(t)$, were modeled using an $\alpha$ function, $g(t)=g_{\max } \times e / \tau_{1}$ $\times \exp \left(-t / \tau_{1}\right)$, for the rising phase and a double exponential, $g(t)=g_{\max } / 2 \times \exp \left(-t / \tau_{2}\right)+$ $g_{\max } / 2 \times \exp \times\left(-t / \tau_{3}\right)$, for the decay. We found this combination of curves most closely fit the actual measured conductances. The peak magnitudes and time constants for the simulated input conductances (Table 1) were set using the average values from the 10 cells recorded in the present study. Because the model cell exhibited a higher resting conductance than that seen in our recordings, we increased the peak magnitudes for $g_{\mathrm{E}}$ and $g_{\mathrm{I}}$, maintaining their relative values, until simulated responses to a single stimulus matched those seen in the data. The temporal features of the simulated conductances were set by normalizing each individual in vivo trace to the average peak magnitude and then visually adjusting the time constants to obtain curves that closely approximated the actual data (see Fig. 5A). To simulate unbalanced adaptation of inhibition, the $g_{\max }$ values for $g_{\mathrm{I}}$ were adjusted as indicated here. For the simulation, $V_{\mathrm{E}}$ and $V_{\mathrm{I}}$ were set to $0 \mathrm{mV}$ and $-80 \mathrm{mV}$, respectively.

Histology. Neurobiotin-filled cells were processed using cyanine 3 as described previously (Higley and Contreras, 2005) and imaged using confocal microscopy $(40 \times, 1.25$ numerical aperture objective; TCS 4D system; Leica, Nussloch, Germany). Single-plane projections were assembled from stacks of images. Brain slices from the contralateral hemisphere were processed for cytochrome oxidase reactivity as described previously (Wilent and Contreras, 2004) and photographed using an Olympus BX51 microscope (Olympus America, Melville, NY).

\section{Results}

We recorded intracellularly from 31 regular spiking neurons in L4 of the barrel cortex of 19 isoflurane-anesthetized rats. Mean resting $V_{\mathrm{m}}$ was $-69.7 \pm 5.4 \mathrm{mV}$ and mean resting input resistance was $34.1 \pm$ 5.6 $\mathrm{M} \Omega$. Cells were located at a cortical depth between 500 and $850 \mu \mathrm{m}$ and had PSP onset latencies of $<5.5 \mathrm{~ms}$ (range, $4.0-5.5 \mathrm{~ms}$ ), corresponding to L4 neurons receiving monosynaptic thalamic input. The short onset latencies suggest that all cells in the present study were located within L4 barrels, as L4 septal cells exhibit PSP onset latencies $>10 \mathrm{~ms}$ (Brecht and Sakmann, 2002). During recording, neurons were filled with neurobiotin for histological verification of depth. We recovered 14 of 31 neurons, including both

A

C barrels in $L 4$.
Table 1. Summary of model parameters

\begin{tabular}{|c|c|c|c|c|}
\hline & \multicolumn{2}{|l|}{ Control } & \multicolumn{2}{|c|}{ Balanced adaptation } \\
\hline & $g_{\mathrm{E}}$ & $g_{1}$ & $g_{\mathrm{E}}$ & $g_{1}$ \\
\hline$g_{\max }$ & $40 \mathrm{nS}$ & $120 \mathrm{nS}$ & $20 \mathrm{nS}$ & $60 \mathrm{nS}$ \\
\hline$T_{1}$ & $1.9 \mathrm{~ms}$ & $2.2 \mathrm{~ms}$ & $3.2 \mathrm{~ms}$ & $3.4 \mathrm{~ms}$ \\
\hline$T_{2}$ & $2.5 \mathrm{~ms}$ & $5.0 \mathrm{~ms}$ & $5.0 \mathrm{~ms}$ & $10.0 \mathrm{~ms}$ \\
\hline$T_{3}$ & $12.5 \mathrm{~ms}$ & $25.0 \mathrm{~ms}$ & $25.0 \mathrm{~ms}$ & $50.0 \mathrm{~ms}$ \\
\hline Onset & $4.5 \mathrm{~ms}$ & $6.5 \mathrm{~ms}$ & $5.0 \mathrm{~ms}$ & $8.0 \mathrm{~ms}$ \\
\hline
\end{tabular}
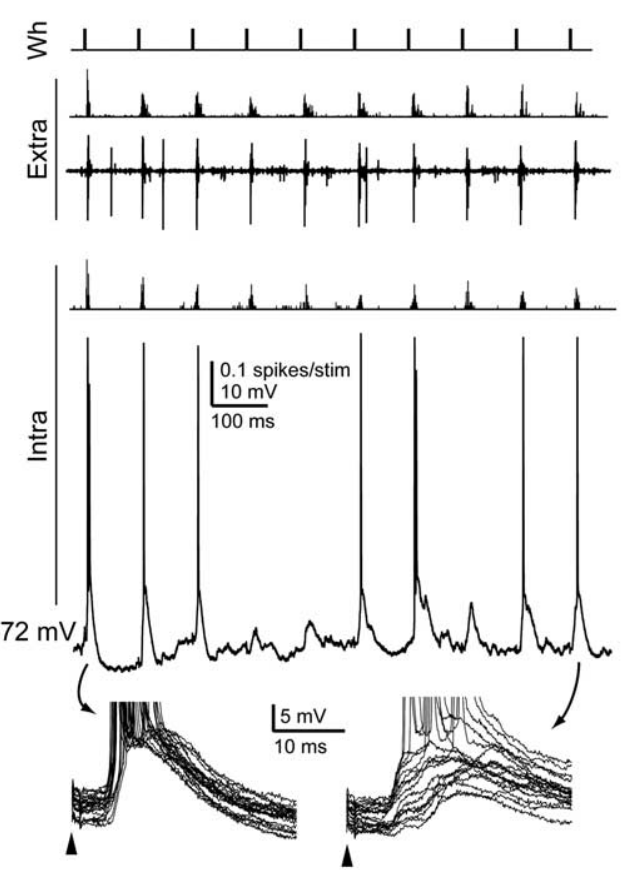

E
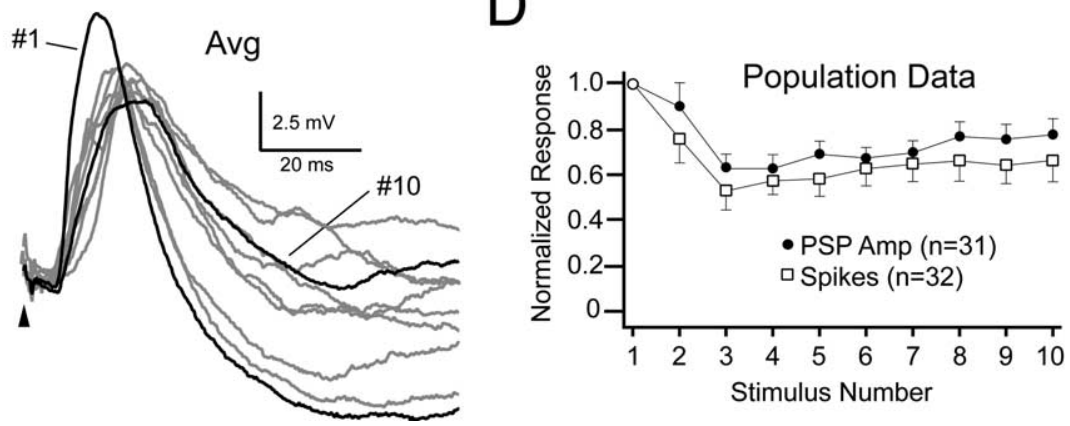

Figure 1. Adaptation of synaptic and spike responses to $10 \mathrm{~Hz}$ PW deflection in L4 barrel cortex neurons. $\boldsymbol{A}$, Single-unit recording (Extra), example trace, and corresponding PSTH (1 ms bins) are shown. Stimulus times are indicated above (Wh). The depth of the recording was $830 \mu \mathrm{m} . \boldsymbol{B}$, Intracellular recording (Intra) from a different animal, example trace, and corresponding PSTH are shown. Resting $V_{\mathrm{m}}$ was $-72 \mathrm{mV}$. The depth of the cell was $510 \mu \mathrm{m}$. Details below, indicated by arrows, are 30 superimposed traces for the first and 10th deflection in the train. Stimulus times are indicated by arrowheads. $\boldsymbol{C}$, Average (Avg) synaptic responses to the first through 10th deflection for the cell in $\boldsymbol{B}$. First (\#1) and 10th (\#10) responses are highlighted in black. D, Mean PSP amplitude (Amp) for all intracellular recordings $(n=31)$ and mean spike output for all cells with suprathreshold responses (intracellular plus single unit recordings; $n=32$ ), normalized to the magnitude of the first response. Error bars indicate \pm SEM. $\boldsymbol{E}$, Reconstruction of a $\mathrm{L} 4(500-850 \mu \mathrm{m})$ spiny stellate and pyramidal neuron recorded from a single micropipette penetration. An additional layer 3 pyramid, recorded in the same track, is shown. The inset shows a lower magnification image of the contralateral hemisphere that was processed for cytochrome oxidase; dashed lines indicate depth of darkly stained

spiny stellate and pyramidal cells. No differences were observed in the evoked responses between groups, and all data were pooled for additional analysis. Figure $1 E$ shows examples of a L4 spiny stellate cell and an L4 pyramidal cell recorded in a single micropipette track. The figure also shows a supragranular pyramidal cell 


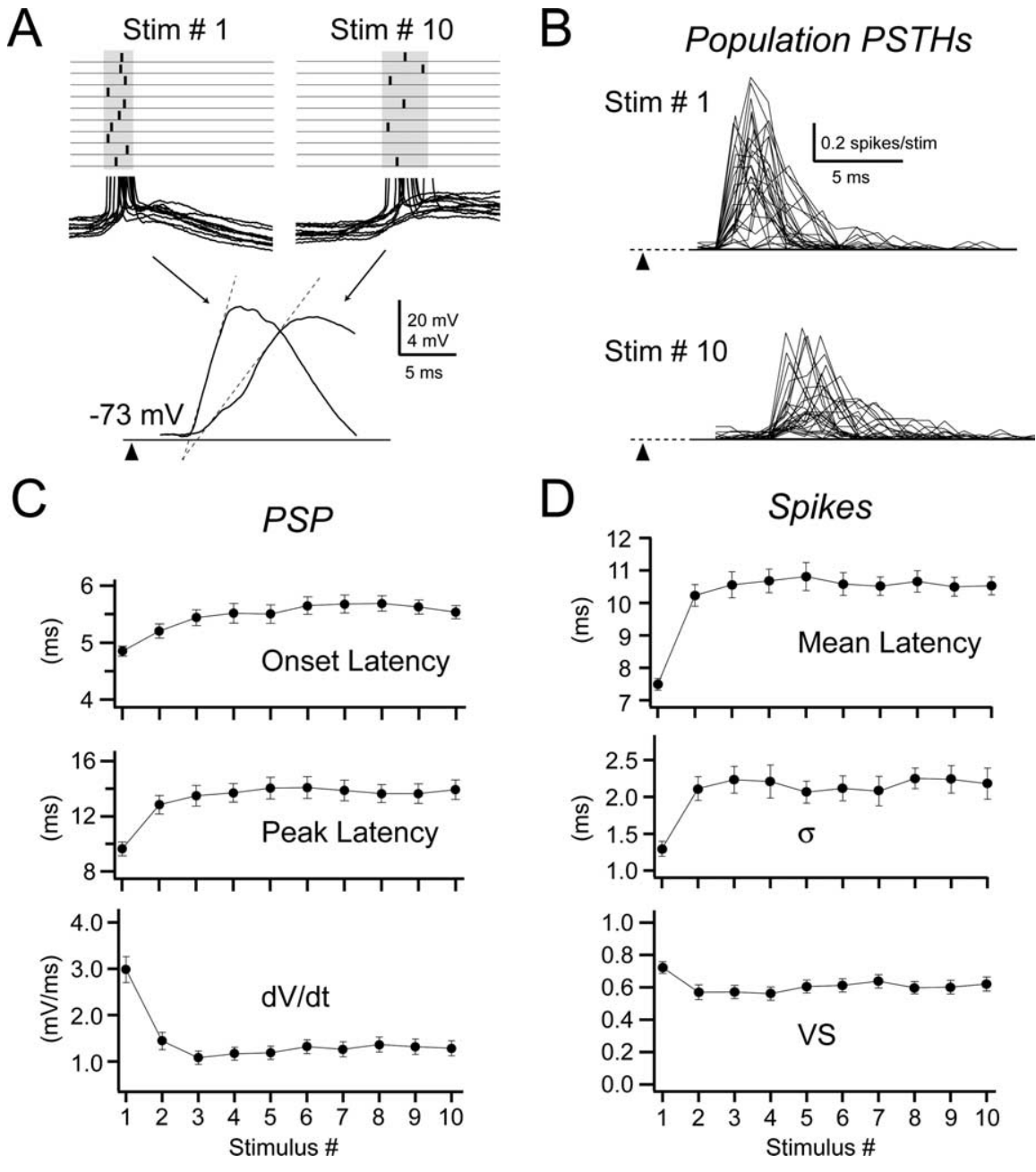

Figure 2. Temporal changes in the synaptic and spike responses during frequency adaptation. $A$, Synaptic response of an $L 4$ neuron $(660 \mu \mathrm{m}$ ) to the first (Stim \#1) and 10th (Stim \#10) PW deflection in a $10 \mathrm{~Hz}$ train. The top traces are 10 sequential synaptic responses, and raster plots illustrate corresponding spike output. The bottom traces are the average synaptic responses from 30 deflections. Resting $V_{\mathrm{m}}$ was $-73 \mathrm{mV}$. Stimulus time is indicated by an arrowhead. Dashed lines are the $10-90 \%$ slope $(d V / d t)$ of the PSPs. B, Population spike output for the first (Stim \#1) and 10th (Stim \#10) PW deflection. 0verlaid traces are the PSTHs (1 ms bins) for all cells with suprathreshold responses. Stimulus time is indicated by arrowheads. $C$, Mean onset latency, peak latency, and $d V / d t$ for all synaptic responses. Error bars indicate \pm SEM. D, Mean latency, SD $(\sigma)$, and vector strength (VS) for all spike responses.

in the same track that was not included in the present analysis. To confirm that L4 corresponds to depths of 500-850 $\mu \mathrm{m}$, the inset illustrates a lower magnification image of the contralateral barrel cortex stained for cytochrome oxidase. L4 barrels are indicated by the darkened staining and are highlighted by the dashed lines.

For all cells, PW deflection evoked a PSP from resting $V_{\mathrm{m}}$ consisting of an initial fast-rising depolarization that often evoked a single spike or less often spike doublets and that was quickly quenched by a longer-lasting (50-100 ms) hyperpolarization. Fifty-five percent of the cells recorded with pipettes containing the control solution of potassium acetate exhibited suprathreshold responses. We also made extracellular recordings from 20 single units, located at similar depths as the intracellular recordings. All extracellular spike waveforms were $>0.7 \mathrm{~ms}$ in duration and had first-spike latencies $<6 \mathrm{~ms}$, corresponding to thalamocortical-receiving L4 regular-spiking units (Simons, 1978; Armstrong-James et al., 1993; Bruno and Simons, 2002). For all measures of spike output, we analyzed separately data from intracellular and extracellular recordings (data not shown).
No differences were found, and therefore, suprathreshold data from all recordings were combined.

Frequency adaptation of spike output and synaptic responses in $\mathrm{L} 4$

We studied the frequency adaptation of L4 whisker-evoked responses by deflecting the PW at $10 \mathrm{~Hz}$ with $1 \mathrm{~s}$ trains. Example traces of a single extracellular unit and an intracellular recording are shown in Figure $1, A$ and $B$, respectively. As illustrated by the corresponding PSTHs, both cells reliably fired action potentials in response to the first whisker deflection in the train (1.2 and 1.6 spikes/stimulus, respectively). The magnitude of the suprathreshold response to the 10th deflection decreased to 0.9 spikes/stimulus (extracellular) and 0.7 spikes/stimulus (intracellular). Figure $1 D$ shows the average spike output across the stimulus train, normalized to the magnitude of the first response, for all cells with suprathreshold responses $(n=32)$ (open squares). The reduction in spike counts was significant, with the mean spike output to the 10th deflection decreased to $67.3 \pm 7.4 \%$ of the first response (Student's paired $t$ test; $p<0.001)$.

Intracellular recordings enabled us to characterize the synaptic changes occurring during frequency adaptation. For the cell in Figure $1 B$, responses to the first deflection in the train showed little variation in amplitude and rate of rise across trials (Fig. $1 B$, bottom left detail). In contrast, responses to the last deflection were more variable (Fig. $1 B$, bottom right detail). The average synaptic responses ( $n=30$ trials) showed a $29.6 \%$ reduction of the PSP amplitude, from 11.5 to $8.1 \mathrm{mV}$ (Fig. $1 C$, first and 10th responses highlighted in bold). This change was accompanied by a broadening of the PSP, a reduction in the rate of rise, and a decrease in the amplitude of the delayed hyperpolarization. The reduction in PSP amplitude was significant for the population of recorded cells $(n=31)$, decreasing to $78 \pm 7 \%$ $(p<0.05)$ of the first response by the 10th deflection (Fig. $1 D$, filled circles).

Repetitive whisker deflection also resulted in changes in the temporal features of the evoked response. Figure $2 \mathrm{~A}$ illustrates the synaptic responses (overlaid traces) and spike output (rasters) to the first and 10th deflection over 10 sequential presentations of a $10 \mathrm{~Hz}$ train for a different cell. We quantified spike timing using three measures: the mean spike latency as well as the $\mathrm{SD}$ and vector strength of individual spike times. The SD gives the exact temporal scatter of evoked spikes, whereas the vector strength provides a normalized value ranging from 0 to 1 of the phase-locking of spikes to a particular latency (see Materials and Methods). Spikes to the first stimulus were highly reliable (1.1 spikes/stimulus) and occurred within a brief poststimulus window as measured by the SD $(\sigma=1.0 \mathrm{~ms})$ and the vector strength (VS, 0.77). Spikes to the 10th stimulus were less reliable $(0.6$ 
spikes/stimulus) and occurred within a slightly broader window $(\sigma=2.0 \mathrm{~ms} ; \mathrm{VS}, 0.70)$. Additionally, spike responses shifted from a mean latency of $7.1 \mathrm{~ms}$ for the first deflection to $11.8 \mathrm{~ms}$ for the 10th. Adaptation encompassed corresponding changes in the timing of the underlying PSP (Fig. $2 \mathrm{~A}$, bottom traces). Across the stimulus train, the average synaptic response for this cell exhibited an increase in onset latency from 4.2 to $4.9 \mathrm{~ms}$ and a larger increase in latency to peak from 9.1 to $16.1 \mathrm{~ms}$. The increased latency to peak coupled with a reduction in peak amplitude gave rise to a decrease in the average rising slope of the PSP of 50\% from 2.9 to $1.5 \mathrm{mV} / \mathrm{ms}(d V / d t)$ (Fig. $2 A$, indicated by the dashed lines).

We quantified the changes in response timing for the population. Figure $2 B$ illustrates the overlaid PSTHs for the first and 10th stimuli of all cells with suprathreshold responses. In addition to a decrease in total spike output (Fig. 1D), mean spike latency for the population increased significantly from $7.5 \pm 0.2$ to $10.5 \pm 0.3 \mathrm{~ms}$ (Fig. $2 D$, top graph) $(p<0.001)$. The mean SD of the spike times also increased from $1.3 \pm 0.1$ to $2.2 \pm 0.2 \mathrm{~ms}$ (Fig. $2 D$, middle graph) $(p<0.001)$, and the mean vector strength decreased $14 \%$ from $0.72 \pm 0.04$ to $0.62 \pm 0.04$ (Fig. $2 D$, bottom graph) $(p<0.05)$. Although we used a shorter poststimulus window than did previous authors for the calculation of vector strength (see Materials and Methods), our values were similar to the proportional change found previously (Khatri et al., 2004). For the synaptic responses, mean onset latency increased from $4.8 \pm 0.1$ to $5.5 \pm 0.1 \mathrm{~ms}$ (Fig. $2 C$, top graph) $(p<$ $0.001)$, latency to peak increased from $9.6 \pm 0.5$ to $13.9 \pm 0.7 \mathrm{~ms}$ (Fig. $2 C$, middle graph) $(p<0.001)$, and $d V / d t$ decreased from $3.0 \pm 0.3$ to $1.3 \pm 0.2 \mathrm{mV} / \mathrm{ms}$ (Fig. $2 C$, bottom graph) $(p<$ $0.001)$. In summary, the data indicate that frequency adaptation in layer 4 results in a slower and smaller synaptic response, which produces weakened and delayed output. Despite a small decrease in spike precision, spike output remained confined to a narrow poststimulus time window.

\section{Relative adaptation of excitatory and inhibitory synaptic conductances}

Although the observed decrease in PSP amplitude and spike output might be explained by a simple withdrawal of excitatory input, such as thalamocortical synaptic depression, the reduction in the delayed hyperpolarization (Fig. 1C) suggests a simultaneous weakening of inhibition. To determine whether repetitive whisker stimulation differentially alters the excitatory and inhibitory inputs to L4 neurons, we recorded 10 cells with the sodium channel blocker QX-314 (25 $\mathrm{mM}$ ) in the pipette. QX-314 also partially blocks calcium and potassium channels (Nathan et al., 1990; Perkins and Wong, 1995; Talbot and Sayer, 1996). However, in previous studies without QX-314, we found that the $V_{\mathrm{m}}$ behaved linearly over the ranges studied here (Higley and Contreras, 2003, 2005), suggesting these voltagedependent currents do not play a large role in mediating whiskerevoked responses. Spikes were typically eliminated within $10 \mathrm{~min}$ of cell penetration, after which $V_{\mathrm{m}}$ and input resistance remained stable for the duration of the recording. This allowed us to measure synaptic responses while altering the $V_{\mathrm{m}}$ of the cell over a wide range via DC current injection through the recording pipette.

An example of this method is shown in Figure $3 A$, where the average response to PW deflection in a L4 neuron is shown at five different $V_{\mathrm{m}}$ levels. By plotting the $V_{\mathrm{m}}$ value against the injected current corrected by the capacitative current $\left(I_{\text {inj }}-I_{\text {Cap }}\right.$; see Materials and Methods), we calculated the total membrane conductance at each time point during the response. For all cells in the present study, no rectification of the current-voltage relationship was observed over the ranges of $V_{\mathrm{m}}$ studied (Fig. $3 B$ ), suggesting our derivation of membrane conductance was not contaminated by voltage-dependent processes. This calculation is illustrated for two points in Figure 3B: baseline (black squares) and near the peak value for evoked synaptic conductance (gray squares). The inverse slope of each line gives the value of the total membrane conductance at that time. Subtraction of the baseline conductance from the total membrane conductance yields the synaptic conductance during the response. Furthermore, the intersection of each line calculated during the response with the line calculated at the baseline gives the apparent synaptic reversal potential at that time. Plots of total synaptic conductance (black trace) and apparent reversal potential (blue trace) for all points in the response are shown in Figure $3 C$. Because the calculation for reversal potential is not reliable for the initial portion of the response where there is little deviation from baseline $V_{\mathrm{m}}$, this interval is shown as a dashed line. The synaptic response exhibited an early peak in the apparent reversal potential reaching $0 \mathrm{mV}$ that coincided with a small increase in synaptic conductance, corresponding to an initial depolarization from all $V_{\mathrm{m}}$ levels and consistent with an excitatory ionotropic glutamatergic (AMPA) synaptic input. This early excitation was followed by a larger increase in synaptic conductance to $53.2 \mathrm{nS}$ (130\% of the resting conductance, $40.9 \mathrm{nS}$ ) that coincided with an abrupt decrease in the apparent reversal potential to $-60 \mathrm{mV}$, consistent with $\mathrm{GABA}_{\mathrm{A}^{-}}$ mediated inhibition. The time point corresponding to the gray squares in Figure $3 B$ is indicated (arrow).

Measuring the apparent reversal potential and total synaptic conductance allowed us to dissect the contributions of excitatory and inhibitory conductances to the response (see Materials and Methods), also shown in Figure $3 C$ (green and red traces, respectively). After whisker deflection, excitation preceded inhibition, consistent with monosynaptic thalamocortical input rapidly followed by feedforward disynaptic inhibition. The much stronger inhibition (peak inhibitory conductance, $40.5 \mathrm{nS}$; peak excitatory conductance, $14.2 \mathrm{nS}$ ) accounted for $\sim 75 \%$ of the total peak synaptic conductance and quickly overtook the excitation. The calculated reversal potential reached $0 \mathrm{mV}$ immediately after the synaptic and excitatory conductances began to rise (Fig. 3C, inset, left vertical line). The reversal potential then hyperpolarized as the inhibitory conductance began (Fig. 3C, inset, right vertical line). Similar results were found for all cells recorded with QX314 , where the mean peak conductances were $16.2 \pm 3.9$ and $43.7 \pm 4.4 \mathrm{nS}$ for excitation and inhibition, respectively. The data revealed that the duration of whisker-evoked excitation typically lasts much longer than the duration of the observed suprathreshold responses (Fig. $2 \mathrm{~B}$ ), confirming that local inhibition is critical in limiting neuronal output from L4.

To study the relative frequency adaptation of excitation and inhibition, we deflected the PW in trains of $10 \mathrm{~Hz}$ while holding the cell at multiple $V_{\mathrm{m}}$ levels via current injection (Fig. $4 \mathrm{~A}$ ). The average responses to the first and 10th deflection are shown in detail below (Fig. $4 B$ ). The color-coded $V_{\mathrm{m}}$ levels are offset to highlight the $V_{\mathrm{m}}$-dependence of the PSP shape because of different reversal potentials for the synaptic components comprising the response. Although the cell was filled with QX-314, early in the recording session, a small number of spikes were evoked, and the rasters and corresponding PSTHs of these suprathreshold responses are shown. As with the example in Figure $2 \mathrm{~A}$, frequency adaptation from the first to the 10th stimulus resulted in fewer spikes (0.6-0.5 spikes/stimulus) that occurred at longer latency $(5.7-7.5 \mathrm{~ms})$ but with only a submillisecond change in the spike window $(\sigma=0.2-0.6 \mathrm{~ms} ; \mathrm{VS}, 0.98-0.90)$. Below the 


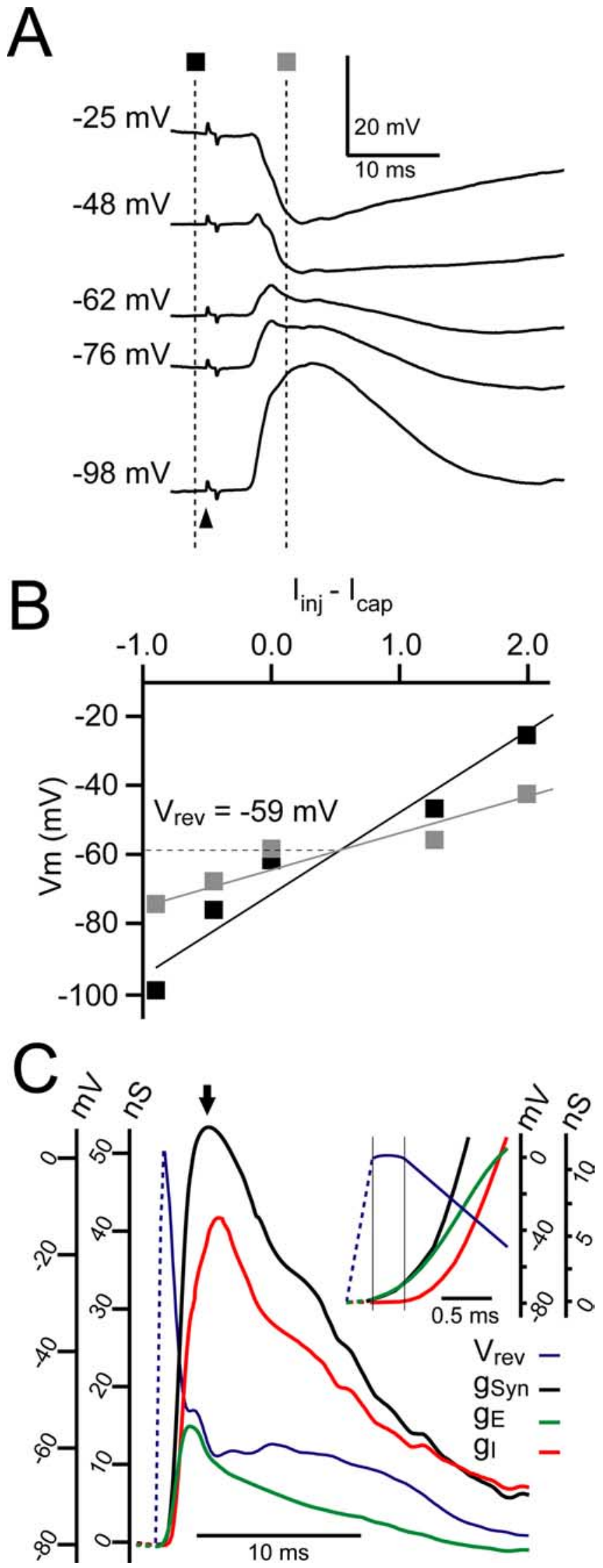

Figure 3. Contribution of excitatory and inhibitory conductances to the PW-evoked synaptic response. $\boldsymbol{A}$, Response to PW deflection of an L4 neuron $(680 \mu \mathrm{m})$ filled with QX-314 and recorded at multiple $V_{m}$ levels via current injection through the recording pipette. Stimulus time is indicated by an arrowhead. $\boldsymbol{B}$, Plot of $V_{m}$ versus injected current corrected for capacitative
PSTHs in Figure 4 are the traces of total synaptic (black), excitatory (green), and inhibitory (red) conductances. Color-coded circles indicate the onset times for excitatory and inhibitory conductances. For this cell, frequency adaptation resulted in a decrease in both excitatory (16.8-9.7 nS) and inhibitory (50.2-30.6 $\mathrm{nS}$ ) conductance over the course of the stimulus train (resting conductance was $32.9 \mathrm{nS}$ ). Furthermore, for both excitation and inhibition, there was an increase in the onset latency $(4.0-5.0 \mathrm{~ms}$ and $4.6-6.3 \mathrm{~ms}$, respectively) and the latency to peak $(5.5-7.2 \mathrm{~ms}$ and $6.3-8.6 \mathrm{~ms}$, respectively).

We quantified the relative adaptation of excitatory and inhibitory conductances for the population of cells studied with QX$314(n=10)$. Reduction of the magnitude of excitatory and inhibitory inputs was balanced over repetitive whisker deflection (Fig. $4 C$, left, filled triangles), with peak excitation decreasing to $52 \pm 6 \%(p<0.001)$ and peak inhibition decreasing to $48 \pm 6 \%$ $(p<0.001)$ of their respective first-deflection magnitudes. The proportional change in excitation and inhibition was not significantly different across cells (paired $t$ test). To assess the relative balance of excitation and inhibition across individual cells, we also plotted the normalized peak magnitude of excitation versus inhibition for the responses to the 10th stimulus (Fig. $4 D$, left). The dashed lines indicate unity $\pm 20 \%$, demonstrating that adaptation of conductances for each cell remained balanced within this margin. In addition to a reduction in magnitude, both excitation and inhibition exhibited increases in onset latency (from $4.6 \pm 0.2$ to $5.2 \pm 0.1 \mathrm{~ms}, p<0.005$ and from $5.1 \pm 0.2$ to $6.5 \pm$ $0.2 \mathrm{~ms}, p<0.001$, respectively) (Fig. $4 C$, right, open squares) and latency to peak (from $6.5 \pm 0.3$ to $8.4 \pm 0.3 \mathrm{~ms}, p<0.001$ and from $7.3 \pm 0.3$ to $9.9 \pm 0.6 \mathrm{~ms}, p<0.001$, respectively) (Fig. $4 C$, right, filled circles). The onset latency increase for inhibition was significantly greater than for excitation $(p<0.001)$.

A comparison of the PSTHs and synaptic responses in Figure $4 B$ revealed that the window for suprathreshold responses (gray boxes) corresponded to the PSP width measured at -50 $\mathrm{mV}$. At this $V_{\mathrm{m}}$, the depolarization is expected to consist exclusively of excitation (rather than reversed inhibition). Thus, we took the PSP width at $-50 \mathrm{mV}$ as an approximate measure for the window of potential spike output in cells recorded with QX-314. In Figure 4D (right), we plotted this value against the "excitation dominance window," taken as the interval between excitation onset and peak inhibition, for responses to the first (open circles) and 10th (open squares) stimuli in all cells. The parameters were well correlated (Fig. $4 D$, solid line) (Pearson's correlation; $r^{2}=0.62$ ). The average values of PSP width (2.2 and $4.0 \mathrm{~ms}$ ) and excitation dominance window (2.6 and $4.9 \mathrm{~ms}$ ) for the first and 10th stimuli, respectively, are shown as filled symbols. Notably, these average values indicate temporal windows, the durations of which are approximately twice the SDs of spike times shown in Figure $2 D(2.6 \mathrm{~ms}$ and $4.4 \mathrm{~ms}$, first and 10th stimuli, respectively), strongly suggesting that the timing of whisker-evoked spike output in L4 can be explained

$\leftarrow$

current $\left(I_{\text {inj }}-I_{\text {cap }}\right.$; see Materials and Methods) for the time points in the response indicated in A. Inverse slope of the best-fit line through each set of points gives the total membrane conductance. Intersection of the line calculated during the response (gray squares) with the line calculated from baseline $V_{m}$ (black squares) gives the apparent synaptic reversal potential $\left(V_{\text {rev }}\right) . C$, Calculated apparent reversal potential (blue trace) and total synaptic (black trace), excitatory (green trace), and inhibitory (red trace) conductances for the PW-evoked response from $A$. The inset highlights the changes in reversal potential and conductances early in the response. 
precisely by the relative magnitude and timing of the excitatory and inhibitory synaptic conductances.

\section{Simulation of evoked responses with balanced and unbalanced adaptation of excitation and inhibition}

The balanced adaptation of the magnitude and timing of excitation and inhibition was surprising given the multiple independent sources of inputs to L4 neurons. To further explore the importance of this balance in shaping L4 output, we simulated a L4 cortical neuron using a singlecompartment model that included in vivolike background activity and Hodgkin and Huxley-type fast conductances (see Materials and Methods). Our goal was not to distinguish the specific mechanisms underlying balanced adaptation but to characterize phenomenologically the sensitivity of spike output magnitude and timing to changes in the relative amounts of synaptic excitation and inhibition. Therefore, we modeled sensory-evoked responses using one excitatory and one inhibitory conductance representing the combined evoked synaptic input to the cell. The simulated input conductances were matched to our in vivo data. Figure $5 A$ illustrates the excitatory and inhibitory conductances (bold traces) used to simulate synaptic inputs for the first and 10th stimuli overlaying the individual conductance traces from the 10 cells in the present study, normalized to the peak magnitudes for the simulated traces.

The simulated whisker-evoked responses are depicted in Figure $5 B$. The top histograms show the total spike output of the model for 100 sequential stimuli, whereas the middle traces illustrate 20 representative $V_{\mathrm{m}}$ responses. The bottom traces depict the excitatory (green) and inhibitory (red) conductances used to generate the data. The left panel shows the response to the first stimulus in a train, which evoked 0.89 spikes/stimulus with a mean spike latency of $7.1 \mathrm{~ms}$. The SD of spike latencies was 1.9 $\mathrm{ms}$, and the vector strength was 0.86 . We then simulated the evoked response under conditions of balanced adaptation for excitation and inhibition. The second panel shows the control response to the 10th stimulus in a train where both excitation and inhibition have been reduced to $50 \%$ of the first stimulus magnitude. In this case, the spike response decreased to 0.70 spikes/ stimulus with an increase in latency to $9.2 \mathrm{~ms}$. There was also a small increase in the spike SD to $2.4 \mathrm{~ms}$, and the vector strength decreased to 0.69 . These values are similar to those obtained from the in vivo data and suggest that the model accurately captures the basic elements of evoked responses of L4 neurons to both single and repetitive stimuli. We then explored the consequences of unbalanced adaptation in the model. The third panel shows the case in which adaptation of excitation is maintained at control levels (50\%), but inhibition is reduced to $25 \%$ of the first stimulus magnitude. Under these conditions, spike output increased to 1.1 spikes/stimulus. Furthermore, the window for spike generation increased sharply $(\sigma=3.6 \mathrm{~ms} ; \mathrm{VS}, 0.43)$. This outcome was a
C
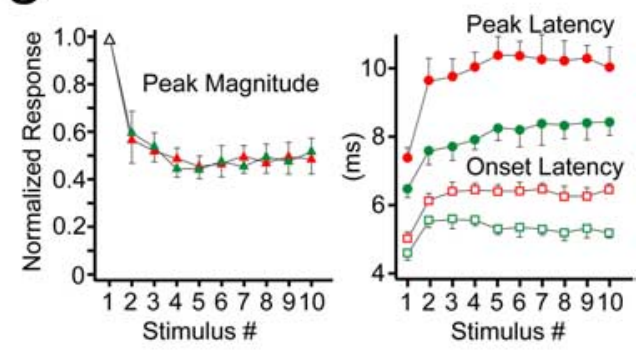

.
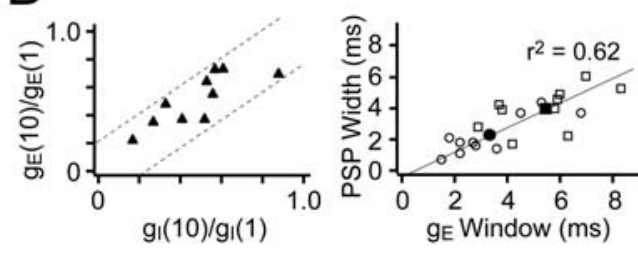

Figure 4. Balanced frequency adaptation of excitatory and inhibitory conductances. $\boldsymbol{A}$, Response to $10 \mathrm{~Hz} \mathrm{PW}$ deflection of an L4 neuron $(800 \mu \mathrm{m})$ filled with QX-314 and recorded at multiple $V_{\mathrm{m}}$ levels. $\boldsymbol{B}$, Response to the first and 10th deflection from $\boldsymbol{A}$. 作 trangles) and inhibitory (red filled triangles) conductances during $10 \mathrm{~Hz}$ train, normalized to the magnitude of the first response, unity $\pm 20 \%$. Right, Plot of PSP width, measured from $-50 \mathrm{mV}$, versus the excitation dominance window, measured as the interval from excitation onset to peak inhibition.

result of the inability of inhibition to suppress longer-latency spikes generated by the unbalanced excitatory drive. The fourth panel illustrates the case in which inhibition is reduced to $75 \%$ of the first stimulus magnitude. Under these conditions, inhibition rapidly quenched the excitatory response, and spike output was reduced to 0.57 spikes/stimulus, although spikes occurred in a narrower window ( $\sigma=1.2 \mathrm{~ms}$; VS, 0.93). Figure $5 C$ shows the data for the spikes/stimulus (filled circles), SD (filled triangles), and vector strength (open squares) versus the percentage of inhibitory conductance relative to the first stimulus magnitude. The graph indicates that the relative amount of inhibition is directly related to spike precision and inversely related to the magnitude of spike output. This finding suggests that, during frequency adaptation, the balanced change in excitation and inhibition allows cortical cells to preserve a narrow window for spike output with a moderate reduction in response magnitude.

\section{Discussion}

Our central finding was that spike output in response to both single and periodic whisker deflection is critically shaped by the dynamic interaction of excitation and inhibition. We have shown that frequency adaptation involves a balanced reduction in excitatory and inhibitory synaptic conductances, resulting in a reduction in total spike output without substantial degrading of the narrow window for spike timing.

Cortical response adaptation has been observed in a variety of sensory systems after visual (Ohzawa et al., 1982), auditory (Shu 


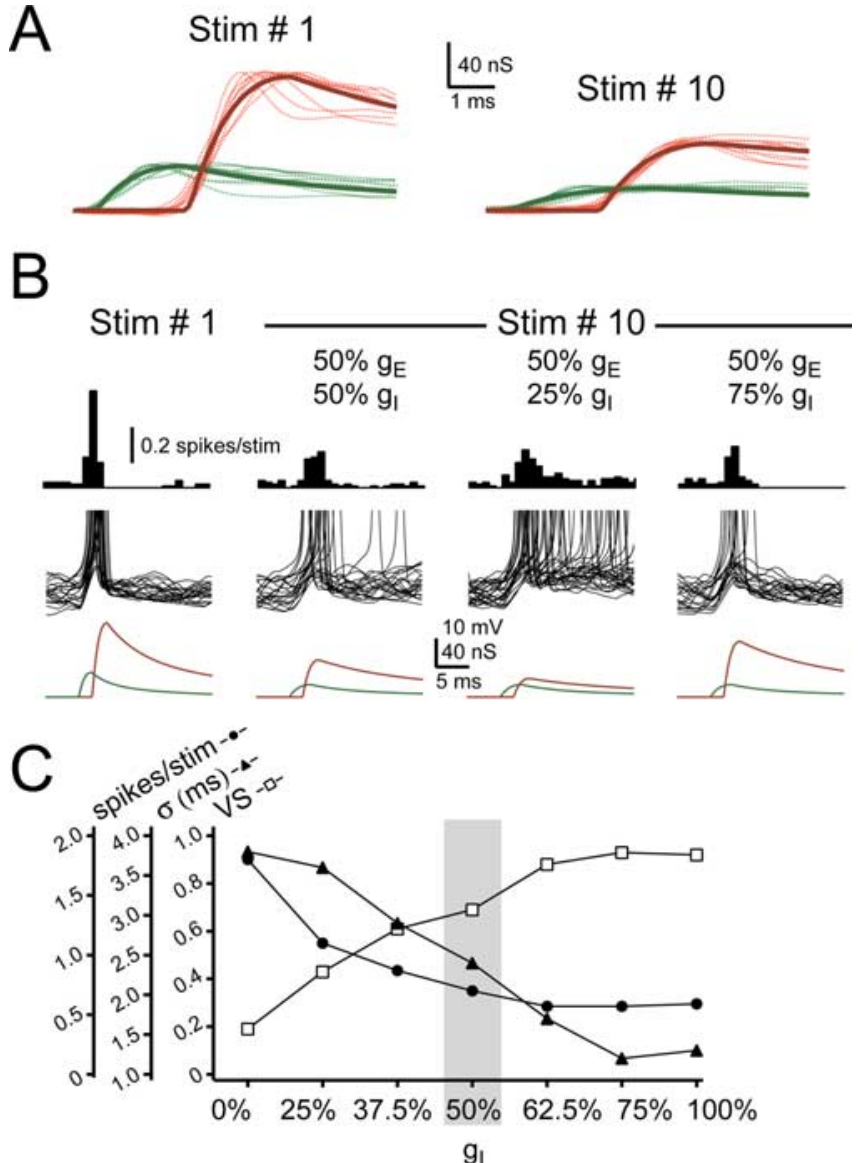

Figure 5. Simulated responses of an $L 4$ neuron to balanced and unbalanced adaptation of excitation and inhibition. $\boldsymbol{A}$, Parameters for simulated excitatory and inhibitory conductances were obtained from the data. Thin traces are individual traces of excitatory (green) and inhibitory (red) conductances to the first (Stim \#1) and 10th (Stim \#10) deflections in a train, taken from the data. Traces are normalized to the average peak amplitude. Overlaid thick traces are the simulated conductances used in the model. $\boldsymbol{B}$, Simulated synaptic and spike responses of the model cell to the first (Stim \#1, left panel) and 10th (Stim \#10, 3 right panels) PW deflections in a $10 \mathrm{~Hz}$ train. The top histograms illustrate spike output for 100 stimuli, middle traces illustrate 20 example synaptic responses, and bottom traces illustrate $g_{\mathrm{E}}$ and $g_{\mathrm{l}}$ used to generate the responses. Responses to the 10th deflection were simulated under three conditions: (1) balanced adaptation of both excitatory and inhibitory conductances to $50 \%$ of the first stimulus magnitude, (2) increased adaptation of inhibition to $25 \%$ of the first stimulus magnitude, and (3) reduced adaptation of inhibition to $75 \%$ of the first stimulus magnitude. C, Plot of spikes/ stimulus (filled circles), SD (filled triangles), and vector strength (open squares) for responses to the simulated 10th stimulus under varying conditions of adaptation of inhibition, expressed as a percentage of first stimulus magnitude. In all cases, adaptation of excitation was $50 \%$ of first stimulus magnitude.

et al., 1993; Wehr and Zador, 2005), and somatosensory stimuli (Hellweg et al., 1977; Simons, 1985; Gardner et al., 1992). Functionally, adaptation of response magnitude to a sustained stimulus has been suggested as a mechanism for reducing cortical gain, matching neuronal sensitivity to input conditions (Ohzawa et al., 1982; Abbott et al., 1997; Carandini et al., 2002). In the present study, we found that PW deflection at $10 \mathrm{~Hz}$ resulted in a $33 \%$ decrease in spike output. This value represents comparable adaptation to that seen in previous work (Simons, 1985; Khatri et al., 2004), although less than that observed by others (Chung et al., 2002; Garabedian et al., 2003). These differences may reflect the methods and depths of anesthesia used across studies. CastroAlamancos (2004) demonstrated that the amount of adaptation was inversely correlated with the level of arousal, suggesting that deeper anesthesia results in greater response adaptation.
As with previous findings (Garabedian et al., 2003; Khatri et al., 2004), repetitive PW deflection produced a significant increase in the mean spike latency. We also observed a significant but submillisecond reduction in spike precision, consistent with reports that repetitive whisker deflection reduces response magnitude without greatly degrading the phase-locking of spike output (Khatri et al., 2004). Moreover, the spike output in response to both single and periodic stimuli occurred within a window that is shorter than the membrane time constant of barrel cortex neurons in vivo, which ranged from 5 to $12 \mathrm{~ms}$ in the present study (data not shown). Thus, the increased spike jitter may not constitute a functional loss of precision.

Accompanying the adaptation of spike output were corresponding changes in the underlying synaptic response, including a $22 \%$ decrease in PSP amplitude. As with the spike data, this value is less than that reported previously (Chung et al., 2002). However, our study differs from previous studies in limiting our recordings to neurons in L4. This distinction is important, because other studies have found differences in adaptation across layers (Ahissar et al., 2000, 2001). We also observed a 57\% reduction in the PSP slope that may explain the increased SD of spike times, because spike precision has been shown to correlate inversely with transient $d V / d t$ (Mainen and Sejnowski, 1995; Axmacher and Miles, 2004). The decrease in slope is similar to that observed in previous studies of cross-whisker suppression, in which preceding whisker deflection caused a reduced magnitude and $d V / d t$ of a subsequent synaptic response because of withdrawal of input to the cell (Higley and Contreras, 2003, 2005).

The similar reduction in spike output and PSP amplitude was somewhat surprising given the nonlinearity of $V_{\mathrm{m}}$ behavior imposed by spike threshold. However, this finding is partially explained by the increased variability in PSP amplitude for the 10th versus first stimulus in a train (Fig. $1 B$ ). Thus, although the average PSP amplitude was reduced, a number of individual trials remained near control amplitude, resulting in less reduction in spike output than might have been observed if all individual PSPs were reduced to the average value. In addition, spike output is generally considered to be a monotonic saturating function of $V_{\mathrm{m}}$ (Koch, 1999). With the high-velocity stimuli used in the present study, it is likely that we are far to the right on such a spike frequency- $V_{\mathrm{m}}$ curve (Wilent and Contreras, 2004), resulting in small reductions in spike output for a given reduction in PSP amplitude.

Using methods similar to those described in the visual (Marino et al., 2005; Priebe and Ferster, 2005) and auditory (Wehr and Zador, 2003, 2005; Zhang et al., 2003) systems, we showed that PW deflection evoked a short-latency excitatory conductance that was rapidly overtaken by a much larger inhibitory conductance. This finding is consistent with the canonical view of L4 activity where thalamocortical excitation triggers strong disynaptic feedforward inhibition (Agmon and Connors, 1991; Swadlow and Gusev, 2000; Porter et al., 2001; Swadlow, 2003; Staiger et al., 2004). The temporal window established between the onset of excitation and the peak of inhibition closely agreed with the excitatory PSP width and the duration of suprathreshold responses observed in spiking neurons, suggesting that the timing of spike output in L4 is a direct consequence of the dynamic interaction between excitatory and inhibitory inputs. A similar sequence of excitation followed by inhibition also plays a role in shaping spike timing in the auditory cortex (Wehr and Zador, 2003), hippocampus (Pouille and Scanziani, 2001), cerebellum (Mittmann et al., 2005), and thalamus (Blitz and Regehr, 2005). Our present findings, combined with this accumulated 
data, strongly indicate that feedforward inhibition is a general mechanism used across brain regions to regulate the precision of spike timing.

Synaptic inputs to L4 originate from corticocortical and thalamocortical sources (Porter et al., 2001; Beierlein et al., 2002, 2003; Bruno and Simons, 2002; Schubert et al., 2003). Numerous studies in vitro have shown that local excitatory and inhibitory synapses between L4 neurons depress at the frequency studied here (Thomson and West, 1993; Petersen, 2002; Beierlein et al., 2003; Cowan and Stricker, 2004; Staiger et al., 2004). Furthermore, thalamocortical synaptic inputs to both excitatory and inhibitory neurons also exhibit short-term depression (Gil et al., 1997; Gibson et al., 1999; Beierlein et al., 2002; Castro-Alamancos and Oldford, 2002). Chung et al. (2002) showed that frequency adaptation in the barrel cortex in vivo is partially dependent on thalamocortical synaptic depression. However, this work did not address whether a reduction in thalamic input might differentially impact the activity of local excitatory and inhibitory circuits. Our experiments demonstrated that repetitive PW deflection resulted in a balanced reduction in the magnitude of excitatory and inhibitory postsynaptic conductances. This result is consistent with a recent extracellular study showing L4 putative excitatory and inhibitory units exhibited similar amounts of frequency adaptation (Khatri et al., 2004). Together, these data indicate that adaptation results from a reduction in total synaptic drive to L4 cortical neurons rather than shift in the relative contribution of excitation versus inhibition. Our findings further suggest that cortical circuits are calibrated to maintain an appropriate balance of excitation and inhibition despite changes in input magnitude, such as occurs with thalamocortical synaptic depression. This conclusion is supported by recent findings that spontaneous cortical activity maintains a strong proportionality of excitatory and inhibitory conductances, even during large fluctuations in total input (Shu et al., 2003).

In addition to a reduction in magnitude, we observed a small increase in onset latency for excitation $(0.6 \mathrm{~ms})$, consistent with previous reports of minimal change in the mean latency of thalamocortical spike output after repetitive PW deflection (Hartings and Simons, 1998; Ahissar et al., 2000; Sosnik et al., 2001; Khatri et al., 2004). We also observed a larger increase in onset latency for inhibition (1.4 ms), suggesting that interneurons in L4 also experience increases in spike latency. This conclusion is supported by previous extracellular studies that found slightly larger increases in the earliest spike latency of putative cortical interneurons versus thalamic units [Khatri et al. (2004), their Fig. 5]. The latency to peak increased for both excitatory and inhibitory conductances, resulting in a slight broadening of the "excitation dominance window" that was correlated with an increased PSP width and agreed with the increased duration of spike output. Thus, frequency adaptation allows a direct test and confirmation of the hypothesis that the interplay of excitatory and inhibitory inputs to L4 neurons directly and precisely determines the window for spike timing. Simulated whisker-evoked responses further suggested that L4 neurons are optimized to exhibit moderate response adaptation without substantial loss of spike precision. Nevertheless, cortical circuits may have the ability to promote either response magnitude or precision at the expense of the other depending on local network conditions. Whether this trade-off occurs in vivo remains to be determined.

One remaining unknown is the function of the narrow spike window established by the dynamic interaction of excitatory and inhibitory conductances. Others have proposed that L4 neurons act as temporal contrast detectors, responding preferentially to highly synchronized thalamic input (Kyriazi and Simons, 1993; Pinto et al., 2000, 2003). In this view, L4 functions as a gate to other cortical layers, filtering out nonoptimal inputs such as whisker deflections with low velocity (Wilent and Contreras, 2004), nonpreferred direction (Kyriazi et al., 1996), or of nonprincipal whiskers (Kyriazi et al., 1996; Fox et al., 2003). Our findings strongly support the proposed view of L4 function and argue that this role is maintained both for single sensory stimuli and for the behaviorally relevant context of repetitive whisking.

\section{References}

Abbott LF, Varela JA, Sen K, Nelson SB (1997) Synaptic depression and cortical gain control. Science 275:220-224.

Agmon A, Connors BW (1991) Thalamocortical responses of mouse somatosensory (barrel) cortex in vitro. Neuroscience 41:365-379.

Ahissar E, Arieli A (2001) Figuring space by time. Neuron 32:185-201.

Ahissar E, Sosnik R, Haidarliu S (2000) Transformation from temporal to rate coding in a somatosensory thalamocortical pathway. Nature 406:302-306.

Ahissar E, Sosnik R, Bagdasarian K, Haidarliu S (2001) Temporal frequency of whisker movement. II. Laminar organization of cortical representations. J Neurophysiol 86:354-367.

Armstrong-James M, Welker E, Callahan CA (1993) The contribution of NMDA and non-NMDA receptors to fast and slow transmission of sensory information in the rat SI barrel cortex. J Neurosci 13:2149-2160.

Axmacher N, Miles R (2004) Intrinsic cellular currents and the temporal precision of EPSP-action potential coupling in CA1 pyramidal cells. J Physiol (Lond) 555:713-725.

Beierlein M, Fall CP, Rinzel J, Yuste R (2002) Thalamocortical bursts trigger recurrent activity in neocortical networks: layer 4 as a frequencydependent gate. J Neurosci 22:9885-9894.

Beierlein M, Gibson JR, Connors BW (2003) Two dynamically distinct inhibitory networks in layer 4 of the neocortex. J Neurophysiol 90:2987-3000.

Blitz DM, Regehr WG (2005) Timing and specificity of feed-forward inhibition within the LGN. Neuron 45:917-928.

Brecht M, Sakmann B (2002) Dynamic representation of whisker deflection by synaptic potentials in spiny stellate and pyramidal cells in the barrels and septa of layer 4 rat somatosensory cortex. J Physiol (Lond) 543:49-70.

Bruno RM, Simons DJ (2002) Feedforward mechanisms of excitatory and inhibitory cortical receptive fields. J Neurosci 22:10966-10975.

Carandini M, Heeger DJ, Senn W (2002) A synaptic explanation of suppression in visual cortex. J Neurosci 22:10053-10065.

Carvell GE, Simons DJ (1990) Biometric analyses of vibrissal tactile discrimination in the rat. J Neurosci 10:2638-2648.

Castro-Alamancos MA (2004) Absence of rapid sensory adaptation in neocortex during information processing states. Neuron 41:455-464.

Castro-Alamancos MA, Oldford E (2002) Cortical sensory suppression during arousal is due to the activity-dependent depression of thalamocortical synapses. J Physiol (Lond) 541:319-331.

Chung S, Li X, Nelson SB (2002) Short-term depression at thalamocortical synapses contributes to rapid adaptation of cortical sensory responses in vivo. Neuron 34:437-446.

Cowan AI, Stricker C (2004) Functional connectivity in layer IV local excitatory circuits of rat somatosensory cortex. J Neurophysiol 92:2137-2150.

Destexhe A, Rudolph M, Fellous JM, Sejnowski TJ (2001) Fluctuating synaptic conductances recreate in vivo-like activity in neocortical neurons. Neuroscience 107:13-24.

Fanselow EE, Nicolelis MA (1999) Behavioral modulation of tactile responses in the rat somatosensory system. J Neurosci 19:7603-7616.

Fee MS, Mitra PP, Kleinfeld D (1997) Central versus peripheral determinants of patterned spike activity in rat vibrissa cortex during whisking. J Neurophysiol 78:1144-1149.

Fox K, Wright N, Wallace H, Glazewski S (2003) The origin of cortical surround receptive fields studied in the barrel cortex. J Neurosci 23:8380-8391.

Garabedian CE, Jones SR, Merzenich MM, Dale A, Moore CI (2003) Bandpass response properties of rat SI neurons. J Neurophysiol 90:1379-1391.

Gardner EP, Palmer CI, Hamalainen HA, Warren S (1992) Simulation of motion on the skin. V. Effect of stimulus temporal frequency on the 
representation of moving bar patterns in primary somatosensory cortex of monkeys. J Neurophysiol 67:37-63.

Gibson JR, Beierlein M, Connors BW (1999) Two networks of electrically coupled inhibitory neurons in neocortex. Nature 402:75-79.

Gil Z, Connors BW, Amitai Y (1997) Differential regulation of neocortical synapses by neuromodulators and activity. Neuron 19:679-686.

Hartings JA, Simons DJ (1998) Thalamic relay of afferent responses to 1- to 12-Hz whisker stimulation in the rat. J Neurophysiol 80:1016-1019.

Hellweg FC, Schultz W, Creutzfeldt OD (1977) Extracellular and intracellular recordings from cat's cortical whisker projection area: thalamocortical response transformation. J Neurophysiol 40:463-479.

Higley MJ, Contreras D (2003) Nonlinear integration of sensory responses in the rat barrel cortex: an intracellular study in vivo. J Neurosci 23:10190-10200.

Higley MJ, Contreras D (2005) Integration of synaptic responses to neighboring whiskers in rat barrel cortex in vivo. J Neurophysiol 93:1920-1934.

Hines ML, Carnevale NT (1997) The NEURON simulation environment. Neural Comput 9:1179-1209.

Khatri V, Hartings JA, Simons DJ (2004) Adaptation in thalamic barreloid and cortical barrel neurons to periodic whisker deflections varying in frequency and velocity. J Neurophysiol 92:3244-3254.

Koch C (1999) Biophysics of computation. New York: Oxford UP.

Kyriazi HT, Simons DJ (1993) Thalamocortical response transformations in simulated whisker barrels. J Neurosci 13:1601-1615.

Kyriazi HT, Carvell GE, Brumberg JC, Simons DJ (1996) Quantitative effects of GABA and bicuculline methiodide on receptive field properties of neurons in real and simulated whisker barrels. J Neurophysiol 75:547-560.

Mainen ZF, Sejnowski TJ (1995) Reliability of spike timing in neocortical neurons. Science 268:1503-1506.

Marino J, Schummers J, Lyon DC, Schwabe L, Beck O, Wiesing P, Obermayer K, Sur M (2005) Invariant computations in local cortical networks with balanced excitation and inhibition. Nat Neurosci 8:194-201.

Mittmann W, Koch U, Hausser M (2005) Feed-forward inhibition shapes the spike output of cerebellar Purkinje cells. J Physiol (Lond) 563:369-378

Nathan T, Jensen MS, Lambert JD (1990) The slow inhibitory postsynaptic potential in rat hippocampal CA1 neurones is blocked by intracellular injection of QX-314. Neurosci Lett 110:309-313.

Ohzawa I, Sclar G, Freeman RD (1982) Contrast gain control in the cat visual cortex. Nature 298:266-268.

Perkins KL, Wong RK (1995) Intracellular QX-314 blocks the hyperpolarization-activated inward current Iq in hippocampal CA1 pyramidal cells. J Neurophysiol 73:911-915.

Petersen CC (2002) Short-term dynamics of synaptic transmission within the excitatory neuronal network of rat layer 4 barrel cortex. J Neurophysiol 87:2904-2914.

Pinto DJ, Brumberg JC, Simons DJ (2000) Circuit dynamics and coding strategies in rodent somatosensory cortex. J Neurophysiol 83:1158-1166.

Pinto DJ, Hartings JA, Brumberg JC, Simons DJ (2003) Cortical damping: analysis of thalamocortical response transformations in rodent barrel cortex. Cereb Cortex 13:33-44.
Porter JT, Johnson CK, Agmon A (2001) Diverse types of interneurons generate thalamus-evoked feedforward inhibition in the mouse barrel cortex. J Neurosci 21:2699-2710.

Pouille F, Scanziani M (2001) Enforcement of temporal fidelity in pyramidal cells by somatic feed-forward inhibition. Science 293:1159-1163.

Priebe NJ, Ferster D (2005) Direction selectivity of excitation and inhibition in simple cells of the cat primary visual cortex. Neuron 45:133-145.

Schubert D, Kotter R, Zilles K, Luhmann HJ, Staiger JF (2003) Cell typespecific circuits of cortical layer IV spiny neurons. J Neurosci 23:2961-2970.

Shu Y, Hasenstaub A, McCormick DA (2003) Turning on and off recurrent balanced cortical activity. Nature 423:288-293.

Shu ZJ, Swindale NV, Cynader MS (1993) Spectral motion produces an auditory after-effect. Nature 364:721-723.

Simons DJ (1978) Response properties of vibrissa units in rat SI somatosensory neocortex. J Neurophysiol 41:798-820.

Simons DJ (1983) Multi-whisker stimulation and its effects on vibrissa units in rat SmI barrel cortex. Brain Res 276:178-182.

Simons DJ (1985) Temporal and spatial integration in the rat SI vibrissa cortex. J Neurophysiol 54:615-635.

Sosnik R, Haidarliu S, Ahissar E (2001) Temporal frequency of whisker movement. I. Representations in brain stem and thalamus. J Neurophysiol 86:339-353.

Staiger JF, Flagmeyer I, Schubert D, Zilles K, Kotter R, Luhmann HJ (2004) Functional diversity of layer IV spiny neurons in rat somatosensory cortex: quantitative morphology of electrophysiologically characterized and biocytin labeled cells. Cereb Cortex 14:690-701.

Swadlow HA (2003) Fast-spike interneurons and feedforward inhibition in awake sensory neocortex. Cereb Cortex 13:25-32.

Swadlow HA, Gusev AG (2000) The influence of single VB thalamocortical impulses on barrel columns of rabbit somatosensory cortex. J Neurophysiol 83:2802-2813.

Talbot MJ, Sayer RJ (1996) Intracellular QX-314 inhibits calcium currents in hippocampal CA1 pyramidal neurons. J Neurophysiol 76:2120-2124.

Thomson AM, West DC (1993) Fluctuations in pyramid-pyramid excitatory postsynaptic potentials modified by presynaptic firing pattern and postsynaptic membrane potential using paired intracellular recordings in rat neocortex. Neuroscience 54:329-346.

Wehr M, Zador AM (2003) Balanced inhibition underlies tuning and sharpens spike timing in auditory cortex. Nature 426:442-446.

Wehr M, Zador AM (2005) Synaptic mechanisms of forward suppression in rat auditory cortex. Neuron 47:437-445.

Welker WI (1964) Analysis of the sniffing of the albino rat. Behaviour 22:223-244.

White EL, Rock MP (1981) A comparison of thalamocortical and other synaptic inputs to dendrites of two non-spiny neurons in a single barrel of mouse SmI cortex. J Comp Neurol 195:265-277.

Wilent WB, Contreras D (2004) Synaptic responses to whisker deflections in rat barrel cortex as a function of cortical layer and stimulus intensity. J Neurosci 24:3985-3998.

Zhang LI, Tan AY, Schreiner CE, Merzenich MM (2003) Topography and synaptic shaping of direction selectivity in primary auditory cortex. Nature 424:201-205. 